\title{
Hadronic Origin of Prompt High-energy Emission of Gamma-ray Bursts Revisited: In the Case of a Limited Maximum Proton Energy
}

\author{
Kai Wang ${ }^{1,2,3}$, Ruo-Yu Liu ${ }^{2,4}$ (iD, Zi-Gao Dai ${ }^{1}$ (D), and Katsuaki Asano ${ }^{5}$ (1) \\ ${ }^{1}$ School of Astronomy and Space Science, Nanjing University, Nanjing 210093, People's Republic of China; dzg@nju.edu.cn \\ ${ }_{2}^{2}$ Max-Planck-Institut für Kernphysik, Saupfercheckweg 1, D-69117 Heidelberg, Germany; ruoyu@mpi-hd.mpg.de \\ ${ }^{3}$ Department of Astronomy and Kavli Institute for Astronomy and Astrophysics, Peking University, Beijing 100871, \\ People's Republic of China; kaiwang@pku.edu.cn \\ ${ }^{4}$ Deutsches Elektronen-Synchrotron (DESY), Platanenallee 6, D-15738 Zeuthen, Germany; ruoyu.liu@desy.de \\ 5 Institute for Cosmic Ray Research, The University of Tokyo, 5-1-5 Kashiwanoha, Kashiwa, Chiba 277-8582, Japan; asanok@icrr.u-tokyo.ac.jp \\ Received 2017 November 12; revised 2018 March 7; accepted 2018 March 11; published 2018 April 10
}

\begin{abstract}
The high-energy $(>100 \mathrm{MeV})$ emission observed by the Fermi Large Area Telescope during the prompt phase of some luminous gamma-ray bursts (GRBs) could arise from the cascade induced by interactions between accelerated protons and the radiation field of GRBs. The photomeson process, which is usually suggested to operate in such a hadronic explanation, requires a rather high proton energy $\left(>10^{17} \mathrm{eV}\right)$ for an efficient interaction. However, whether GRBs can accelerate protons to such a high energy is far from guaranteed, although they have been suggested as the candidate source for ultrahigh-energy cosmic rays. In this work, we revisit the hadronic model for the prompt high-energy emission of GRBs with a smaller maximum proton energy than the usually adopted value estimated from the Bohm condition. In this case, the Bethe-Heitler pair production process becomes comparably important or even dominates over the photomeson process. We show that with a relatively low maximum proton energy with a Lorentz factor of $10^{5}$ in the comoving frame, the cascade emission can still reproduce various types of high-energy spectra of GRBs. For most GRBs without high-energy emission detected, the maximum proton energy could be even lower and relax the constraints on the parameters of the GRB jet resulting from the nondetection of GRB neutrinos by IceCube.
\end{abstract}

Key words: cosmic rays - gamma-ray burst: general - radiation mechanisms: non-thermal

\section{Introduction}

High-energy gamma rays have been discovered in a few tens of gamma-ray bursts (GRBs), spanning from the prompt emission phase to the afterglow phase, by the Large Area Telescope (LAT) onboard the Fermi satellite (Ackermann et al. 2013a). The long-lasting LAT emission in the afterglow phase, detected up to thousands of $\mathrm{s}$ or even 1 day after the burst, usually shows a power-law decay with time and may arise from the external forward shock via synchrotron radiation and inverse Compton (IC) scattering of accelerated electrons in forward shocks (Kumar \& Barniol Duran 2009, 2010; Liu et al. 2013; Wang et al. 2013; Fukushima et al. 2017). On the other hand, in many of these GRBs, the high-energy emission during the prompt phase presents a rapid variability and a temporal correlation with the $\mathrm{keV} / \mathrm{MeV}$ emission, implying an internal dissipation origin (Maxham et al. 2011; Tang et al. 2017) rather than an external shock origin. Generally, the GRB spectra should contain three elemental components, i.e., a Band component, a blackbody component, and an extra power-law component extending to high energies (Zhang et al. 2011; Guiriec et al. 2015). For the high-energy emissions, based on their spectra, one has three types. (i) The 090926A-type. The high-energy spectrum is hard (photon index $\sim-1.6$ ) and shows an evident high-energy cutoff (Ackermann et al. 2011), also forming an extra component. (ii) The 090902B-type. The highenergy spectrum is quite flat (photon index $\sim-2$ ) without an obvious high-energy cutoff in the Fermi/LAT energy region (the high-energy cutoff may exist at a higher energy beyond the upper limit of Fermi/LAT; Abdo et al. 2009a), manifesting itself as an extra component relative to the $\mathrm{keV} / \mathrm{MeV}$ one. (iii) The 080916C-type. The high-energy spectrum seems to be consistent with the extrapolation of the empirical Band function from the $\mathrm{keV} / \mathrm{MeV}$ emission (Band et al. 1993; Abdo et al. 2009b). There are also some common features shared by these GRBs, such as the high isotropic energy and large bulk Lorentz factor inferred from observations.

The radiation mechanism of the prompt high-energy emission is still unclear. Both leptonic and hadronic origins have been suggested. The leptonic origin usually relates to the external shock, such as synchrotron radiation of electrons accelerated in an external shock or prompt $\mathrm{keV} / \mathrm{MeV}$ photons upscattered by the accelerated electrons in the external shock (Kumar \& Barniol Duran 2009; Ackermann et al. 2011; Beloborodov et al. 2014). Asano et al. (2010) considered a proton-dominated GRB jet in which protons are accelerated up to $10^{20} \mathrm{eV}$, initiating an electromagnetic (EM) cascade via the photomeson process in the photon field of the jet. These authors found that the intensity of secondary $e^{ \pm}$pairs produced in the cascade can significantly exceed that of the primary electrons at high energies. And their synchrotron radiation and the IC scattering off $\mathrm{keV} / \mathrm{MeV}$ photons in the jet can reproduce the observed high-energy spectrum. Note that the photomeson process has a high threshold energy of $\sim 0.34 \mathrm{GeV}$ for photons (in the rest frame of a proton), which translates to a proton energy of $E_{p}>10^{17}(\Gamma / 300)^{2}\left(\varepsilon_{\gamma, b} / 300 \mathrm{keV}\right)^{-1} \mathrm{eV}$ in the observer's frame, where $\Gamma$ is the bulk Lorentz factor of the jet, and $\varepsilon_{\gamma, b}$ is the break energy in the spectrum of the prompt $\mathrm{keV} / \mathrm{MeV}$ emission. Although the maximum proton energy can easily reach such a high energy when considering the acceleration by a relativistic shock in the Bohm condition, especially for those very energetic GRBs, such a condition may not be achieved in real shocks. What is more, the energy 
dissipation mechanism during the prompt emission phase is actually unclear. Besides the shock acceleration, mechanisms such as neutron decay and magnetic reconnection have also been suggested to account for the conversion from the kinetic energy of the GRB jet to the nonthermal energies of emitting particles (e.g., Rees \& Mészáros 2005; Zhang \& Yan 2011). Thus, the maximum achievable proton energy in the GRB's jet is far from certainty. In the case that the maximum proton energy falls below the threshold energy of the photomeson process to interact with the photons of energy of the spectral peak, the photomeson process may not be efficient to produce the observed high-energy flux in a GRB. On the other hand, the Bethe-Heitler $(\mathrm{BH}) e^{ \pm}$pair production process of protons $\left(p \gamma \rightarrow p e^{+} e^{-}\right)$has a lower threshold energy of about $1 \mathrm{MeV}$, and its energy-loss rate peaks at $\sim 10 \mathrm{MeV}$. Thus, protons may still induce an EM cascade via this process, producing highenergy emission during the prompt emission phase.

In this paper, we revisit the hadronic model for the prompt high-energy emission of GRBs, under the consideration of a relatively limited acceleration ability of protons. Different from the previous numerical studies, we are dedicated to an analytical method that may help us to better understand the physical processes underlying the observed radiation. This paper is organized as follows. In Section 2, the model and method adopted are described, and the results in the benchmark case are presented. We show that the spectrum of the prompt high-energy emission of GRB 090926A, GRB 090902B, and GRB 080916C, which are regarded as representatives of three different types of high-energy spectrum, can be well fitted in the considered case in Section 3. In Section 4, we discuss the possible leptonic contribution and neutrino emission under the considered model, and we summarize this work in Section 5.

\section{Proton-induced Cascade in the GRB Jet}

Let us consider an isotropically expanding shell with the Lorentz factor $\Gamma$ at radius $R$ from the central engine. For simplicity, all physical quantities inside the shell are assumed to be homogeneous. The spectrum of $\mathrm{keV}-\mathrm{MeV}$ photons in the prompt emission phase can usually be depicted by the so-called Band function (Band et al. 1993),

$$
n\left(\varepsilon_{\gamma}\right)=\left\{\begin{array}{l}
A\left(\frac{\varepsilon_{\gamma}}{100 \mathrm{keV}}\right)^{\alpha} \exp \left(-\frac{\varepsilon_{\gamma}}{\varepsilon_{\gamma, 0}}\right), \\
\varepsilon_{\gamma}<(\alpha-\beta) \varepsilon_{\gamma, 0}, \\
A\left(\frac{(\alpha-\beta) \varepsilon_{\gamma, 0}}{100 \mathrm{keV}}\right)^{\alpha-\beta} \exp (\beta-\alpha)\left(\frac{\varepsilon_{\gamma}}{100 \mathrm{keV}}\right)^{\beta}, \\
\varepsilon_{\gamma}>(\alpha-\beta) \varepsilon_{\gamma, 0},
\end{array}\right.
$$

where $\alpha$ and $\beta$ are the low-energy and high-energy photon indexes, respectively, separated by the break energy $\varepsilon_{\gamma, b} \equiv$ $(\alpha-\beta) \varepsilon_{\gamma, 0}$. The normalized coefficient is $A=\Gamma^{2} U_{\gamma} /$ $\left[\int_{\varepsilon_{\gamma, \min }}^{\varepsilon_{\gamma, \max }} n\left(\varepsilon_{\gamma}\right) \varepsilon_{\gamma} d \varepsilon_{\gamma}\right]$, where $U_{\gamma}=L_{\gamma} /\left(4 \pi R^{2} \Gamma^{2} c\right)$ is the photon energy density in the comoving frame and $L_{\gamma}$ is the luminosity integrated from $\varepsilon_{\gamma, \min }$ to $\varepsilon_{\gamma \text {,max }}$, which are fixed to be $1 \mathrm{keV}$ and $10 \mathrm{MeV}$, respectively.

In the outflow, the primary protons are assumed to be accelerated to a power-law distribution in energy space, say, $n_{p}^{\prime}\left(\gamma_{p}^{\prime}\right) \propto \gamma_{p}^{\prime-s}$ for $\gamma_{p, \text { min }}^{\prime} \leqslant \gamma_{p}^{\prime} \leqslant \gamma_{p, \text { max }}^{\prime}$, where $\gamma_{p \text {,min }}^{\prime}$ is
Table 1

The Benchmark Parameters of GRB Outflow

\begin{tabular}{lr}
\hline \hline Parameter & \multicolumn{1}{c}{ Values } \\
\hline$z$ & 1 \\
$\Gamma$ & 300 \\
$s$ & 2 \\
$\alpha$ & -1 \\
$\beta$ & -2.2 \\
$\varepsilon_{\gamma, p}(\mathrm{keV}-10 \mathrm{MeV})$ & $300 \mathrm{keV}$ \\
$L_{\gamma}(1 \mathrm{k})$ & $10^{53} \mathrm{erg} \mathrm{s}^{-1}$ \\
$\epsilon_{p}$ & 10 \\
$\epsilon_{B}$ & 1 \\
$R$ & $10^{14} \mathrm{~cm}$ \\
$\gamma_{p, \max }^{\prime}$ & $10^{5}$ \\
\hline
\end{tabular}

taken to be just slightly larger than unity in the comoving frame and $\gamma_{p \text {, max }}^{\prime}$ is treated as a parameter because of its uncertainty. The energy density of the accelerated protons in the comoving frame of the outflow is linked with the photon density by $U_{p}=\epsilon_{p} U_{\gamma}{ }^{6}$ As relativistic protons are injected into the dense $\mathrm{keV} / \mathrm{MeV}$ photon field of the outflow, the photomeson and $\mathrm{BH}$ processes would operate and produce the secondary gamma-ray photons, $e^{ \pm}$pairs, and neutrinos. For both processes, we adopt a semi-analytical treatment for the emissivity and spectrum of the secondaries, following Kelner \& Aharonian (2008).

In our calculations, the free parameters are the luminosity $L_{\gamma}$ in $1 \mathrm{keV}-10 \mathrm{MeV}$; the bulk Lorentz factor of the outflow $\Gamma$; the dissipation radius $R$; the energy equipartition coefficients for protons $\epsilon_{p}$ and magnetic field $\epsilon_{B}$; the spectral properties of the $\mathrm{keV} / \mathrm{MeV}$ emission, say, spectral index $\alpha, \beta$ and the break energy $\varepsilon_{\gamma, b}$; the redshift of the GRB $z$ and the maximum proton energy (Lorentz factor) in the comoving frame $\gamma_{p \text {,max }}^{\prime}$ (or the Bohm factor); and the spectral index $s$. Although GRBs detected by the LAT during the prompt emission phase usually show a large bulk Lorentz factor, we consider a moderate one, say, $\Gamma=300$, as the benchmark value, since it is a more commonly accepted value for most GRBs. The benchmark values of other parameters can be found in Table 1. Figure 1 presents the ratio between the dynamical timescale of one pulse in the comoving frame of the outflow $t_{\mathrm{dyn}}^{\prime} \simeq R / \Gamma c$ and the energy-loss timescales $t_{\mathrm{c}}^{\prime}$ of relevant processes in the comoving frame as a function of proton energy under the benchmark parameters. These ratios can be regarded as the cooling efficiency of these processes, i.e., $t_{\text {dyn }}^{\prime} / t_{p \gamma}$ for photomeson cooling efficiency, $t_{\mathrm{dyn}}^{\prime} / t_{\mathrm{BH}}$ for $\mathrm{BH}$ cooling efficiency, and $t_{\text {dyn }}^{\prime} / t_{\text {syn }}$ for synchrotron cooling efficiency. Such results shown in Figure 1 are consistent with the estimations in earlier studies (e.g., Crumley \& Kumar 2013; Kumar \& Zhang 2015); i.e., the BH cooling dominates over the photomeson cooling at lower $\gamma_{p}^{\prime}$, while at larger $\gamma_{p}^{\prime}$, the latter is dominant. Note that the energy-loss timescale for the photomeson and $\mathrm{BH}$ processes depends on the cross section of the interaction (denoted by $\sigma$ ) and the fraction of energy lost by the proton in one interaction (i.e., the inelasticity $\kappa$ ), given a fixed number density of the target photon field. The value of the product of these two quantities $\kappa \sigma$ reaches the peak when the photon energy is $0.3 \mathrm{GeV}$ (Mücke et al. 2000) and $10 \mathrm{MeV}$ (Chodorowski et al. 1992) in the

\footnotetext{
6 Note that the definition here is not identical to the normal definition of the energy equipartition coefficient, which is usually the fraction of the dissipated kinetic energy that goes into nonthermal protons.
} 


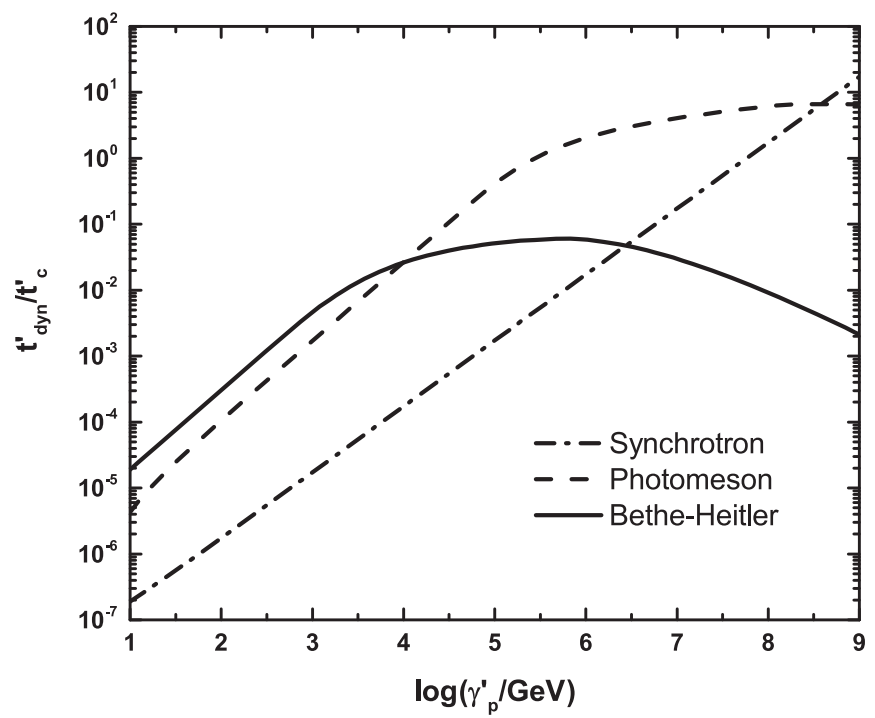

Figure 1. Ratios of the dynamical and cooling timescales of different processes for a proton as a function of the proton's Lorentz factor. All quantities are measured in the comoving frame and calculated with the benchmark parameters for the GRB jet.

rest frame of the proton for the photomeson process and the $\mathrm{BH}$ process, respectively. Thus, for each proton energy, there is a corresponding typical photon energy $\varepsilon_{t}$, at which the interaction or energy loss will be more efficient than that at other energies. We obtain $\varepsilon_{\mathrm{t}} \simeq 1 \mathrm{MeV}(\Gamma / 300)\left(\gamma_{p}^{\prime} / 10^{5}\right)^{-1}(1+z)^{-1}$ for the photomeson process and $\varepsilon_{\mathrm{t}} \simeq 30 \mathrm{keV}(\Gamma / 300)\left(\gamma_{p}^{\prime} / 10^{5}\right)^{-1}(1+z)^{-1}$ for the $\mathrm{BH}$ process. Therefore, for a given spectral break at $\varepsilon_{\gamma, b}=300 \mathrm{keV}$ with $z=1$ in the benchmark case, we can expect a break in the curve of the cooling timescale as a function of $\gamma_{p}^{\prime}$ for the photomeson process around $\gamma_{p}^{\prime}=1.5 \times 10^{5}$ and for the $\mathrm{BH}$ process around $\gamma_{p}^{\prime}=5 \times 10^{3}$, as can be seen in Figure 1 . The second break for the $\mathrm{BH}$ process around $\gamma_{p}^{\prime}=10^{6}$ is due to the low-energy cutoff of the Band spectrum at $\varepsilon_{\text {min }}=1 \mathrm{keV}$, because the corresponding $\varepsilon_{\mathrm{t}}$ for higher-energy protons is even lower than the minimum energy of the target photon field.

In addition to the photomeson and $\mathrm{BH}$ processes, synchrotron radiation can also make protons lose their energies. The synchrotron cooling timescale for a relativistic proton is $t_{s y n}^{\prime}=\frac{9}{4 \gamma_{p}^{\prime}} \frac{m_{p}^{3} c^{5}}{e^{4} B^{2}}$, which is less important than either the photomeson or BH processes for $\gamma_{p}^{\prime}<3 \times 10^{8}$. We ignore the IC cooling of the proton in this work, since it would be dominated by the photomeson cooling at any energies (Asano 2005). Usually, in terms of $\gamma_{p \text {,max }}^{\prime}$, it can be determined by equating the acceleration timescale $t_{\text {acc }}^{\prime}=\mathcal{A} t_{L} \beta_{s}^{-2} \simeq \mathcal{A} \gamma_{p}^{\prime} m_{p} c / e B$ to $\min \left\{t_{\mathrm{dyn}}^{\prime}, t_{c}^{\prime}\right\}$, where $\beta_{s}$ is the speed of the internal shock in units of the speed of light, which is close to unity in the scenario of GRB internal shock; $t_{L}^{\prime}$ is the Larmor gyration timescale; $t_{c}^{\prime}$ is the energy-loss time due to the photomeson, $\mathrm{BH}$, and synchrotron; and $\mathcal{A}(>1)$ is defined as the Bohm factor, which measures the deviation from the acceleration in the Bohm limit. In this work, we are mainly concerned with $\gamma_{p, \max }^{\prime} \leqslant 10^{6}(\mathcal{A} \gg 1)$, where the energy-loss efficiency is smaller than unity. Thus, as an approximation, we consider a constant injection rate of secondary particles from the photomeson and $\mathrm{BH}$ processes during one pulse.
Once produced, the secondary photons and $e^{ \pm}$pairs of sufficiently high energies inevitably initiate an EM cascade in the dense $\mathrm{keV} / \mathrm{MeV}$ photon fields via $\gamma \gamma$ annihilation and IC scattering. High-energy photons can also be produced via synchrotron radiation of $e^{ \pm}$pairs in the strong magnetic field, given $B \simeq 10^{5} \epsilon_{B}^{1 / 2}\left(L_{\gamma} / 10^{53} \mathrm{erg} \mathrm{s}^{-1}\right)^{1 / 2}\left(\mathrm{R} / 10^{14} \mathrm{~cm}\right)^{-1}(\Gamma / 300)^{-1}$ $\mathrm{G}$. For simplicity, the synchrotron radiation of intermediate particles such as charged pions and muons is treated as the suppression factors, respectively, $1-\exp \left(-t_{\pi, \text { syn }}^{\prime}\left(E_{\pi}^{\prime}\right) / \tau_{\pi}^{\prime}\left(E_{\pi}^{\prime}\right)\right)$ and $1-\exp \left(-t_{\mu \text {,syn }}^{\prime}\left(E_{\mu}^{\prime}\right) / \tau_{\mu}^{\prime}\left(E_{\mu}^{\prime}\right)\right)$, which rely on the energy of the parent proton, say, $E_{\pi}=0.2 E_{p}$ and $E_{\mu}=0.15 E_{p}$, where $\tau_{\pi}^{\prime}=2.6 \times 10^{-8} \gamma_{\pi}^{\prime} \mathrm{s}$ and $\tau_{\mu}^{\prime}=2.2 \times 10^{-6} \gamma_{\mu}^{\prime} \mathrm{s}$ are the lifetimes of pions and muons. On the other hand, in the considered energy range $\left(\gamma_{p \text {, max }}^{\prime} \leqslant 10^{6}\right)$, the synchrotron radiation of these intermediate charged particles is very weak compared to the contribution from secondary electrons; hence, their contribution to the overall flux is neglected. The differential density of the cascaded electron (including both $e^{-}$and $\left.e^{+}\right) n_{e}(\gamma)$ is governed by the energy transport equation

$$
\frac{\partial n_{e}^{\prime}}{\partial t}+\frac{\partial}{\partial \gamma_{e}^{\prime}}\left(\dot{\gamma}_{e}^{\prime} n_{e}^{\prime}\right)=Q_{e}+\dot{n}_{e, \gamma \gamma}^{\prime},
$$

where $Q_{e}$ is the injection of electrons from protons via the photomeson or $\mathrm{BH}$ processes, while $\dot{n}_{e, \gamma \gamma}^{\prime}$ represents the injection of electrons from photons via the $\gamma \gamma$ annihilation. We ignore the escape term in the above equation, since electrons can hardly escape such a highly turbulent outflow of GRBs with a strong magnetic field and a very dense photon field. The cascade develops quite quickly, and the distribution of electrons in energy space would reach the quasi-steady state, since the timescales of the relevant processes are typically much shorter than the dynamical timescale $t_{\text {dyn }}^{\prime}$ in the GRBs' environment. Thus, we can derive the electron spectrum from the energy transport equation in the quasi-steady state $\left(\partial n_{e}^{\prime} / \partial t=0\right)$ by

$$
n_{e}^{\prime}\left(\gamma_{e}^{\prime}\right)=-\frac{1}{\gamma_{e}^{\prime}} \int_{\gamma_{e}^{\prime}}^{\infty} d \widetilde{\gamma}_{e}^{\prime}\left[Q_{e}\left(\widetilde{\gamma}_{e}^{\prime}\right)+\dot{n}_{e, \gamma \gamma}^{\prime}\left(\widetilde{\gamma}_{e}^{\prime}\right)\right]
$$

and avoid the time-consuming Monte Carlo simulations of the development of the cascade. Our detailed treatment can be found in the Appendix.

In Figure 2, we show the cascaded electron spectrum in the quasi-steady state with different $\gamma_{p \text {, max }}^{\prime}$. We separate the electrons originating from the $\mathrm{BH}$ process (solid curves) from those originating from the photomeson process (dashed curves). Basically, the spectra from these two processes have a similar structure, with a power law of $\sim \gamma_{e}^{\prime-3}$ at low energies becoming harder at a certain energy and then followed by a cutoff. The low-energy part is mainly determined by the electrons produced in the $\gamma \gamma$ annihilation. At such an energy, the produced $e^{ \pm}$pair approximately shares the energy of the parent photon, and the injected electron spectrum generally follows the spectrum of the high-energy photon provided by a high interaction rate in the outflow, which can be assumed to be of the form $E^{-\Gamma_{\gamma}}$. Based on Equation (3), the power-law index of electrons in the quasi-steady state is then $\Gamma_{e}=\Gamma_{\gamma}+1$. On the other hand, high-energy photons should be produced via either synchrotron or IC radiation by those electrons (the decay of $\pi^{0}$ from the photomeson process is very high and makes a 


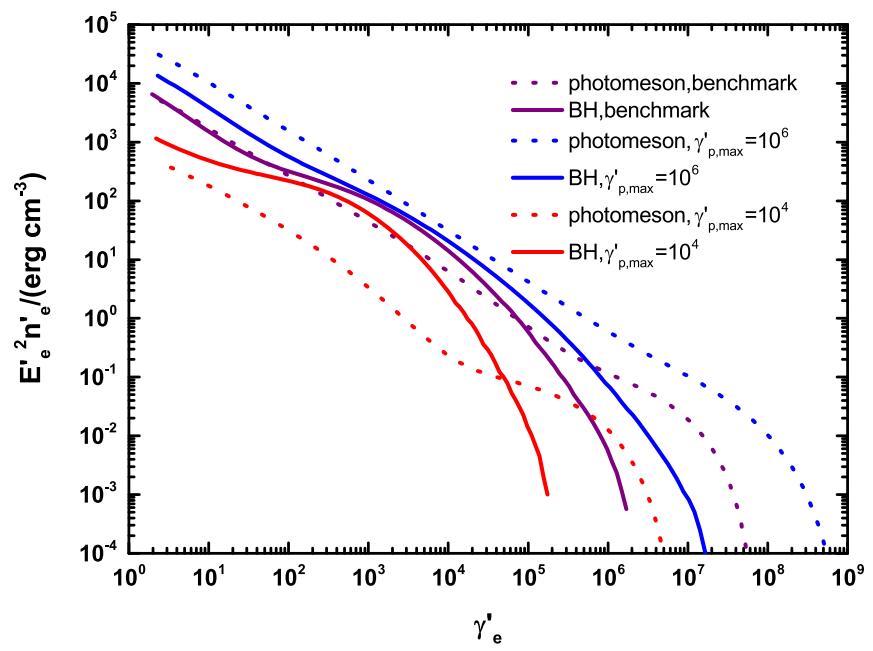

Figure 2. Quasi-steady-state spectrum of the cascaded electrons in the comoving frame of the GRB jet with fiducial parameters (except for the maximum proton Lorentz factor). Solid curves show the electrons originating from the $\mathrm{BH}$ process, while dashed curves show the electrons originating from the photomeson process. Red, purple, and blue lines are for $\gamma_{p, \max }^{\prime}=10^{4}, 10^{5}$, and $10^{6}$, respectively.

negligible contribution of gamma rays at the considered energy here), giving rise to the relation $\Gamma_{\gamma}=\left(\Gamma_{e}+1\right) / 2$. Thus, we obtain $\Gamma_{e}=3$ and $\Gamma_{\gamma}=2$, which is consistent with our results shown in Figures 2 and 3. We note that in some scenarios of the EM cascade (such as the one in the hadronic model for $\mathrm{GeV}$ photons of blazars or the one developed in intergalactic space), a flat electron spectrum $\left(\Gamma_{e}=2\right)$ is expected to appear at the low energy, which is due to the cooling of higher-energy electrons without injection. In the scenario of a GRB jet, however, the injection of electrons from $\gamma \gamma$ annihilations can extend to quite low energies, since the energy of the target photons is high and hence such a segment of the spectrum will not show up. At higher energies, the injection of electrons from the channel of $\gamma \gamma$ annihilation becomes less important due to insufficient high-energy photons, while the photomeson and $\mathrm{BH}$ processes become more efficient. Thus, the electrons injected directly from the interactions of protons cause the hardening of the quasi-steady-state electron spectrum at high energies. The cutoff in the electron spectrum is due to the cutoff in the proton spectrum. Because the energy of secondary electrons produced in the photomeson process is higher than that in the $\mathrm{BH}$ process, the cutoff energy of the electron spectrum from the photomeson process is higher than that from the $\mathrm{BH}$ process.

The spectrum of the cascade emission with different $\gamma_{p \text {, max }}^{\prime}$ is exhibited in Figure 3. In the case of $\gamma_{p \text {,max }}^{\prime}=10^{5}$ or the benchmark case, the contribution of the $\mathrm{BH}$ process is higher than that of the photomeson process below $1 \mathrm{GeV}$. Note that in the benchmark case, the typical energy of electrons producing $1 \mathrm{GeV}$ photons via synchrotron radiation is $\gamma_{e}^{\prime} \simeq 10^{4}$, while the Lorentz factor of the electrons produced directly from the $\mathrm{BH}$ process is typically $\sim 10^{-3}\left(m_{p} / m_{e}\right) \gamma_{p}^{\prime} \simeq \gamma_{p}^{\prime}$. So this energy is consistent with the proton energy below which the $\mathrm{BH}$ process is more efficient than the photomeson process of cooling protons (as shown in Figure 1). The spectral breaks around $100 \mathrm{MeV}$ are due to $\gamma \gamma$ annihilation inside the jet. Note that the photomeson efficiency $\left(\sim t_{\text {dyn }}^{\prime} / t_{p \gamma}^{\prime}\right)$ is about eight times higher

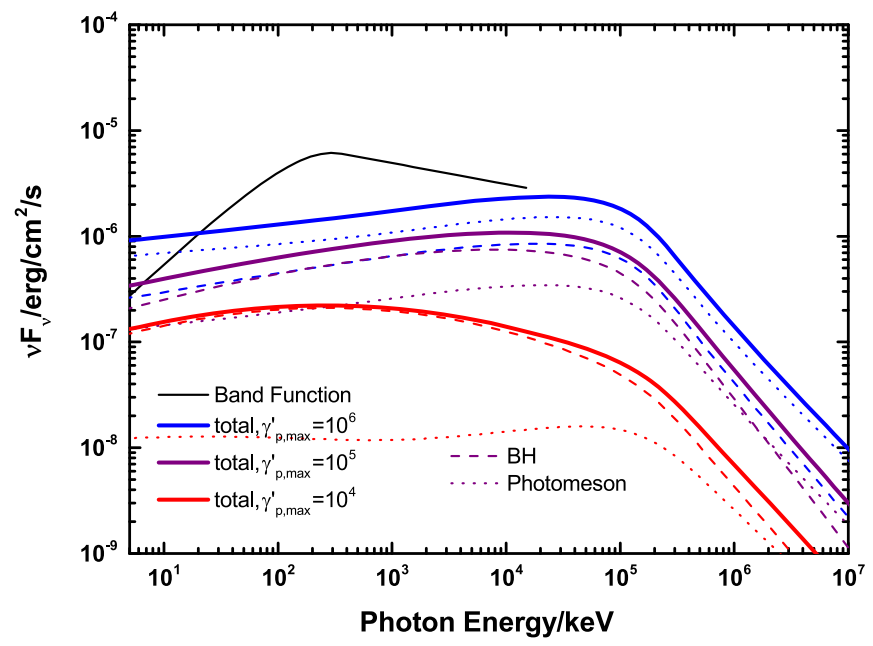

Figure 3. Spectrum of the cascade emissions in a GRB jet with the benchmark parameters (except the maximum proton Lorentz factor $\gamma_{p, \max }^{\prime}$ ). The dashed and dotted curves correspond to the $\mathrm{BH}$ and photomeson processes, respectively.

than the BH efficiency $\left(\sim t_{\mathrm{dyn}}^{\prime} / t_{\mathrm{BH}}^{\prime}\right)$ at $\gamma_{p}=10^{5}$, as can be seen in Figure 1. Considering a proton spectrum with an index $s=2$ and $\gamma_{p, \max }^{\prime}=10^{5}$, the overall photomeson efficiency (integral over the proton spectrum) is still about 4 times higher than the $\mathrm{BH}$ efficiency. After subtracting the energy taken away by the generated neutrinos, the energy of protons lost to EM components from the photomeson process is still about twice that from the $\mathrm{BH}$ process. Thus, one may expect the photomeson process to have a larger contribution here. However, we should keep in mind that the photons we observe are after absorption, while protons with the same energy generally produce higher-energy electrons via the photomeson process than via the $\mathrm{BH}$ process. Before the absorption of highenergy photons due to $\gamma \gamma$ annihilation, the spectrum of the cascade emission from the photomeson process peaks around $\mathrm{TeV}$, while that from the $\mathrm{BH}$ process peaks at several tens of $\mathrm{MeV}$. The peak flux of the former is indeed about twice that of the latter. However, the contribution of the $\mathrm{BH}$ process is more important than that of the photomeson process around $100 \mathrm{MeV}$. After the absorption of the $\mathrm{TeV}$ gamma rays, energy is reprocessed into lower-energy photons (below $\mathrm{MeV}$ ) via synchrotron radiation of the generated secondary electrons. This explains why the $\mathrm{BH}$ process dominates around $100 \mathrm{MeV}$, although its overall efficiency is less than that of the photomeson process.

In Figure 3, we can also see that the flux of the cascade emission is higher with a larger $\gamma_{p \text {, max }}^{\prime}$. This is because the interaction efficiency for a proton increases with the proton's energy, especially for the photomeson process. So a large $\gamma_{p \text {, max }}^{\prime}$ enhances the overall interaction efficiency and, consequently, the flux of the cascade emission. This is the reason that the cascade flux is reduced by a lot in the case of $\gamma_{p \text {, max }}^{\prime}=10^{4}$. What is more, in the case of $\gamma_{p \text {,max }}^{\prime}=10^{4}$, the maximum Lorentz factor of an electron is $\sim 10^{-3} m_{p} / m_{e} \sim \gamma_{p, \max }^{\prime}=10^{4}$. This leads to the softening of the spectrum at a few MeV. Thus, we do not expect that the hadronic model can work well in explaining $\mathrm{GeV}$ emissions with such a low maximum energy for protons and hence do not explore the case of $\gamma_{p \text {,max }}^{\prime}=10^{4}$ in the fitting of the spectrum of LAT GRBs. But this case 


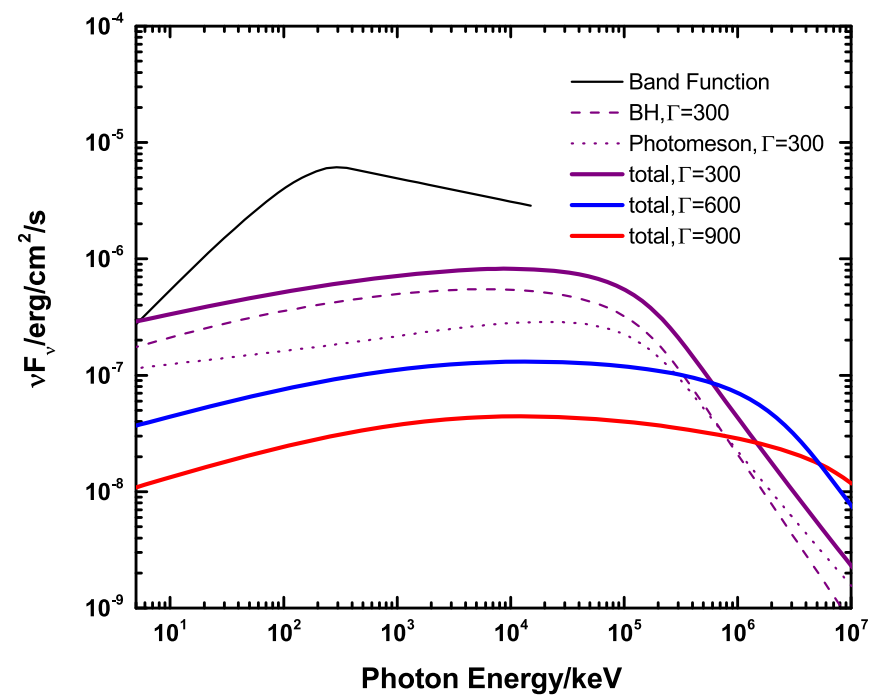

Figure 4. Same as Figure 3, but for a different bulk Lorentz factor of the GRB jet $\Gamma$.

would be a hint for the nondetection of neutrinos, as we will discuss later. In the case of $\gamma_{p \text {,max }}^{\prime} \gtrsim 10^{6}$, the $\mathrm{BH}$ process contributes to the total emission by a few tens of percent, which is consistent with the claim by Asano et al. (2009b). In the figure, each curve for the $\mathrm{BH}$ and photomeson processes is the sum of the synchrotron and IC radiation by the cascaded $e^{ \pm}$ pairs. We note that whether the photomeson process has more contribution than the $\mathrm{BH}$ process does not only depend on $\gamma_{p \text {,max }}^{\prime}$. The spectrum shape of the target photon field in the comoving frame, in terms of the break energy $\varepsilon_{\gamma, b}^{\prime}\left(\simeq \varepsilon_{\gamma, b} / \Gamma\right)$ and low-energy spectral index $\alpha$, also has important influence. In the benchmark case, the break energy is $\varepsilon_{\gamma, b}^{\prime}=2 \mathrm{keV}$ in the comoving frame. If the break energy is higher, the turnover in photomeson efficiency around $\gamma_{p}^{\prime}=10^{5}$ as shown in Figure 1 will shift to lower energy, and hence the photomeson process will dominate even in the case of $\gamma_{p \text {, max }}^{\prime}=10^{5}$ (Gao et al. 2012). On the other hand, if the low-energy spectrum of the Band component is softer (i.e., a smaller $\alpha$ ), the photomeson efficiency will not decrease as fast with energy below the turnover and may still have more contribution than the $\mathrm{BH}$ process.

We also investigate the influence of the bulk Lorentz factor $\Gamma$ and the dissipation radius $R$. The results are shown in Figures 4 and 5. Since the energy density of target photon field has the dependency relationship $U_{\gamma} \propto \Gamma^{-2} R^{-2}$, a larger $\Gamma$ or $R$ results in a lower interaction efficiency. So the flux of cascade emission decreases as $\Gamma$ and $R$ increase, and the cutoff energy due to $\gamma \gamma$ annihilation increases for a larger $\Gamma$ and $R$.

In Figure 6, the effect of the equipartition coefficient for the magnetic field $\epsilon_{B}$, which is defined as the ratio of the magnetic field energy density to the photon energy density in the comoving frame (i.e., $\epsilon_{B} \equiv U_{B} / U_{\gamma}$ ), on the spectrum of the cascade emission is shown. The magnetic field in the comoving frame is then $B=\sqrt{2 \epsilon_{B} L_{\gamma} / \Gamma^{2} R^{2} c}$. Basically, a higher $\epsilon_{B}$ leads to a more important synchrotron radiation, while a smaller $\epsilon_{B}$ leads to a more important IC radiation. Since electrons usually emit higher-energy photons via IC radiation than via synchrotron radiation, the flux of the cascade emission at high energies becomes higher for a smaller $\epsilon_{B}$. On the other hand, more high-energy photons in turn produce more $e^{ \pm}$pairs at

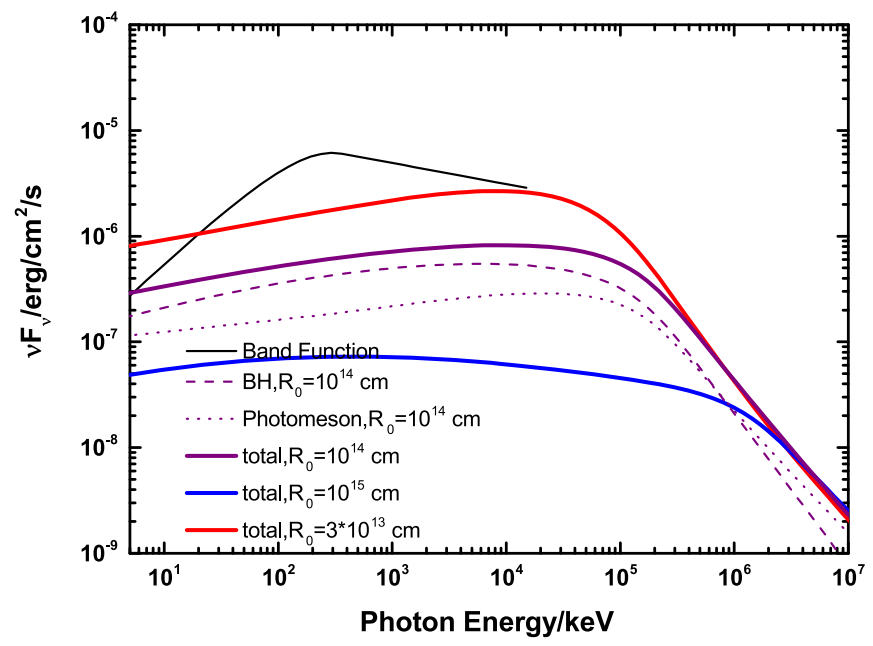

Figure 5. Same as Figure 3, but for a different dissipation radius of the GRB jet $R$.

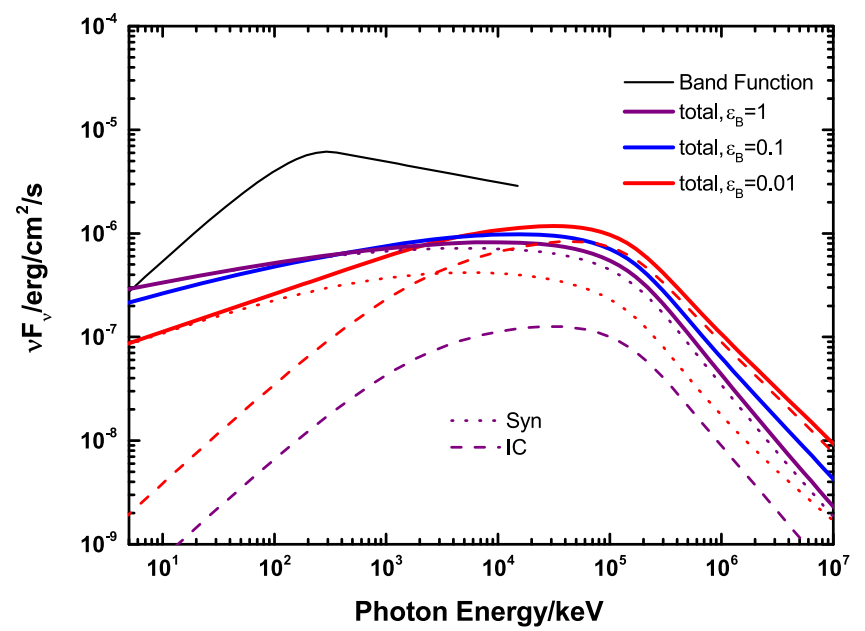

Figure 6. Same as Figure 3, but for the equipartition coefficient for the magnetic field in the GRB jet $\epsilon_{B}$. The dashed and dotted lines correspond to the IC and synchrotron radiation, respectively.

high energies, making a harder electron spectrum than that in the case of a large $\epsilon_{B}$, as is shown in Figure 7. This explains why the spectrum is also harder in the case of $\epsilon_{B}=0.01$ than in the case of $\epsilon_{B}=1$ at $\mathrm{keV}$ energies, where the emission is dominated by synchrotron radiation of these (relatively) lowenergy electrons in both cases. One can see that the gamma-ray flux around $100 \mathrm{MeV}$ is not very sensitive to the value of $\epsilon_{B}$. This is because the injection rate of the secondary electrons that emit $100 \mathrm{MeV}$ photons is dominated by the $\gamma \gamma$-annihilation process (i.e., $\dot{n}_{e, \gamma \gamma}$ in the right-hand side of Equation (3)), mainly relies on the photon field, and does not change with the magnetic field. On the other hand, the jet is already an electron calorimeter even if only considering IC radiation under the benchmark parameters. Therefore, we expect the total photon production rate at a certain energy is roughly equal to the energy injection rate of the electrons that emit these photons (i.e., $L_{\gamma} \sim L_{e}$ ). A large $\epsilon_{B}$ only changes the way the electrons radiate their energy away but does not increase the photon production rate. Although the energy of the gamma-rayemitting electrons is different in the IC-dominated case with a small $\epsilon_{B}$ from that in a synchrotron-dominated case with a large $\epsilon_{B}$, the resultant gamma-ray flux is at the the same level, since 


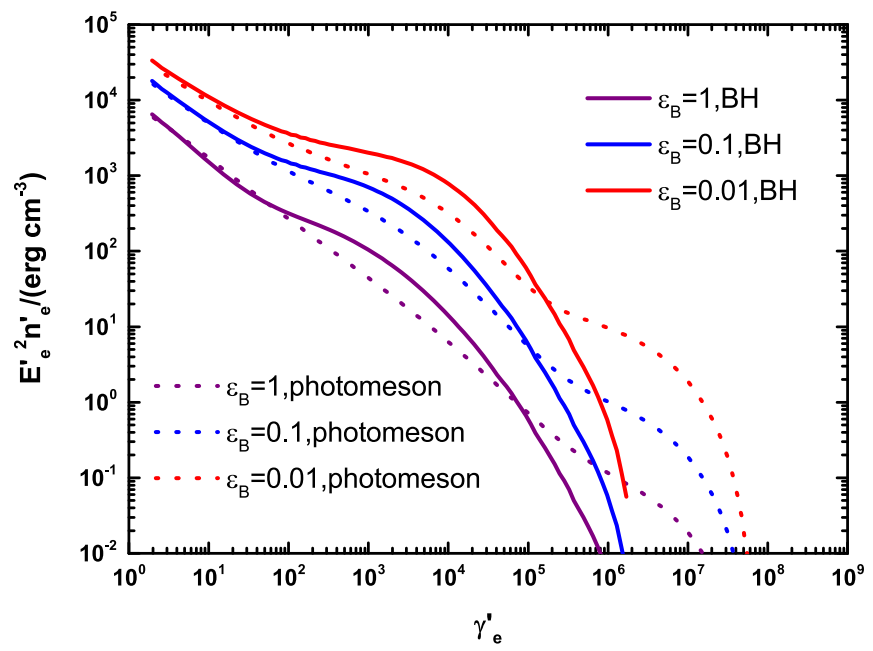

Figure 7. Quasi-steady-state spectrum of the cascaded electrons in the comoving frame of the GRB jet with fiducial parameters (except for the equipartition coefficient of the magnetic field). Purple, blue, and red curves are for $\epsilon_{B}=1,0.1$, and 0.01 , respectively, while solid and dashed curves are the contribution by the $\mathrm{BH}$ and photomeson processes, respectively.

the energy injection rate of electrons in the relevant energy range is roughly constant with respect to electron energy (i.e., $E^{2} \dot{n}_{e, \gamma \gamma} \propto E^{0}$ ).

\section{Application to LAT GRBs}

In this section, we apply our calculations to explain the highenergy spectrum of GRB 090926A, GRB 090902B, and GRB 080916C, which represent three different types of spectral feature. The origin of prompt $\mathrm{keV} / \mathrm{MeV}$ emission is beyond the scope of this work, and we just regard it as a preexisting target photon field for the $\mathrm{BH}$ and photomeson processes. In our calculations below, we employ a flat spectrum for the injected proton spectrum $(s=2)$. Since the efficiencies for both the photomeson and $\mathrm{BH}$ processes increase with proton energy in the considered energy range, the gamma-ray flux (also the neutrino flux) will be lower if we employ a softer proton spectrum, and vice versa. However, the shape of the spectrum of the cascade emission should not change significantly, as the spectrum of secondary $e^{ \pm}$does not depend significantly on the spectrum at injection as long as the cascade has fully developed. Thus, what mainly matters is the baryonic loading factor.

\subsection{GRB 090926A}

GRB 090926A, a luminous long GRB at redshift $z=2.1$, was detected at high energy (above $100 \mathrm{MeV}$ ) by Fermi-LAT during the prompt phase with both a spatial and temporal correlation with the Fermi-Gamma-ray Burst Monitor (GBM) data (Ackermann et al. 2011). The spectrum in the GBM band is selected by a time-integrated spectrum within an interval between $T_{0}+3.3$ and $T_{0}+21.6 \mathrm{~s}$, based on the Band function with the best-fitting parameters of $\alpha=-0.6, \beta=-2.6$, and $\varepsilon_{\gamma, p}=256 \mathrm{keV}$. The high-energy spectrum manifests itself as an extra component in addition to the conventional Band component in both the time-integrated and time-resolved spectra with an evident spectral cutoff around $\mathrm{GeV}$. The peak of the $\mathrm{GeV}$ light curve and that of the Band component are in coincidence, implying a strong correlation for the origin of these photons.
The spectral fit to GBM and LAT data by the Fermi Collaboration is presented by the green dashed lines in Figure 8 . We take the $\mathrm{keV} / \mathrm{MeV}$ component as the target photon and calculate the EM cascade emission induced by the photomeson production and $\mathrm{BH}$ process to see whether it can match the observed high-energy extra component. Due to the hard spectrum (with a photon index -1.72) of the extra component, the IC radiation is supposed to play the leading role around $\mathrm{GeV}$. As a result, a small $\epsilon_{B}$ is expected. We adopt two maximum Lorentz factors of accelerated protons, i.e., $\gamma_{p \text {, max }}^{\prime}=10^{5}$ and $\gamma_{p \text {, max }}^{\prime}=10^{6}$, and the two corresponding Bohm factors are $\mathcal{A} \simeq 830$ and $\mathcal{A} \simeq 67$ for the two groups of parameters adopted in Figure 8, respectively. In the former case, high-energy emission is dominated by the $\mathrm{BH}$ process, while in the latter one, the photomeson process dominates. Both cases can give a good fit to the data and require a high baryonic loading factor $\left(\epsilon_{p}>10\right)$, consistent with the results in previous studies on the hadronic origin of the extra component of GRBs (Asano et al. 2009a, 2009b). Note that a larger proton energy budget is required to be invoked for $\gamma_{p, \max }^{\prime}=10^{5}$ owing to a lower overall interaction efficiency for protons.

Due to the measured cutoff in the high-energy spectrum, the Lorentz factor of the outflow is estimated to be $\Gamma \simeq 720 \pm 76$ in the framework of the internal shock model (Ackermann et al. 2011). In addition, the radius is estimated to be $10^{14}-10^{15} \mathrm{~cm}$. In our fitting, with the radius $R=10^{14} \mathrm{~cm}$ being fixed, a Lorentz factor of $\Gamma=720$ and 800 is adopted to produce a spectrum cutoff for $\gamma_{p, \max }^{\prime}=10^{5}$ and $\gamma_{p, \max }^{\prime}=10^{6}$, respectively, which is consistent with the estimation in Ackermann et al. (2011).

\subsection{GRB 090902B}

GRB 090902B is another intense burst detected at a redshift of $z=1.822$. The joint spectral fit of GBM and LAT data between $T_{0}+4.6$ and $T_{0}+9.6 \mathrm{~s}$ by Abdo et al. (2009a) shows that the spectrum can be described by a Band function of $\alpha=0.07, \beta=-3.9$, and $\varepsilon_{\gamma, p}=908 \mathrm{keV}$ on top of a power law with a photon index -1.94 , with excesses at both high energy ( $>100 \mathrm{MeV}$ ) and low energy (below $50 \mathrm{keV})$. The lowenergy excess is not a unique feature to GRB 090902B, and there are some other GRBs presenting the same feature, e.g., GRB 080319B and GRB 110731A (Asano et al. 2010; Ackermann et al. 2013b). For GRB 090902B, the low-energy excess seems to be consistent with the extrapolation from the high-energy excess and can be reproduced by the cascade emissions induced by the photomeson process along with the high-energy one (Asano et al. 2010). In addition to the target photons from the Band spectrum, the contributions to the target photons from the low-energy excess are taken into account by implementing the iteration.

Since the extra high-energy spectrum extends up to tens of $\mathrm{GeV}$ without a clear cutoff, a lower limit of $\Gamma=1000$ is inferred for the bulk Lorentz factor of the GRB outflow (Abdo et al. 2009a). Such a large $\Gamma$ would lead to a low efficiency for the hadronic processes and requires a high baryonic loading factor to explain the extra component. Similar to the fitting to GRB 090926A, we also adopt $\gamma_{p, \max }^{\prime}=10^{5}$ and $\gamma_{p, \max }^{\prime}=10^{6}$ for GRB 090902B, and the two corresponding Bohm factors are $\mathcal{A} \simeq 3500$ and $\mathcal{A} \simeq 760$ for the two groups of parameters adopted in Figure 9, respectively. As shown in Figure 9, the $\mathrm{BH}$ process dominates in the former case, while the 

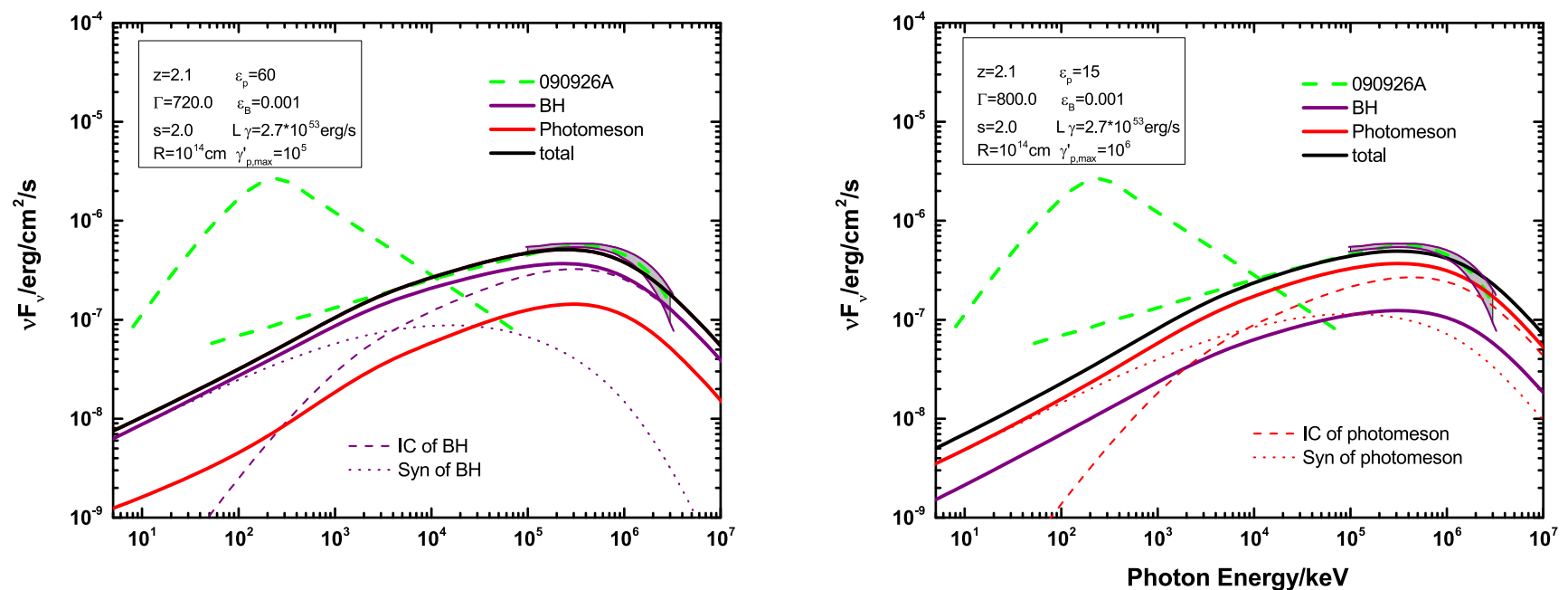

Figure 8. Spectral fitting for GRB 090926A with $\gamma_{p \text {,max }}^{\prime}=10^{5}$ (left) and $\gamma_{p \text {,max }}^{\prime}=10^{6}$ (right). The purple curves represent the emissions of cascaded electrons originating from the BH process, while the red curves represent that from the photomeson process. The red and purple dotted and dashed curves show the synchrotron and IC radiation of the electrons from the dominant process. The green dashed curves show the fitting of the burst's spectrum by the Fermi-LAT collaboration. The shaded region shows the uncertainty in the spectrum fitting at the high-energy end.

photomeson process dominates in the latter case. For the bestfitting parameters, both cases require $\epsilon_{p}>100$, resulting in an isotropic-equivalent proton luminosity beyond $10^{55} \mathrm{erg} \mathrm{s}^{-1}$. Such a large proton luminosity is normally a challenge to the hadronic model. However, since GRBs with extra components are usually very luminous, a high proton luminosity may be possible. On the other hand, the beaming correction may alleviate the energetic problem by a factor of $\theta^{2} / 2$, with $\theta$ being the jet opening angle.

Fixing the dissipation radius at $R=10^{14} \mathrm{~cm}$, we reproduce the high-energy excess by adopting $\Gamma=1100$ and 1500 for $\gamma_{p, \max }^{\prime}=10^{5}$ and $\gamma_{p, \max }^{\prime}=10^{6}$, respectively. In the case of $\gamma_{p, \max }^{\prime}=10^{5}$, the $\mathrm{BH}$ process dominates, and due to a small maximum proton Lorentz factor, the Lorentz factors of secondary $e^{ \pm}$are generally $\lesssim 10^{5}$. Thus, their synchrotron radiation cannot produce $\mathrm{GeV}$ photons, and a small $\epsilon_{B}=0.06$ is invoked to ensure efficient IC emission at $\mathrm{GeV}$. The superposition of synchrotron and IC emission is used to explain the flat extra high-energy component. Although there is finetuning in our fitting, all of the parameters are in their reasonable ranges. For the case of $\gamma_{p, \max }^{\prime}=10^{6}$ (photomeson-dominated case), the Lorentz factors of produced $e^{ \pm}$could reach $10^{8}$. Thus, synchrotron radiation of these electrons can solely account for the extra component, given an appropriate value of $\epsilon_{B}$. The low-energy excess below $50 \mathrm{keV}$ is roughly fitted in both cases, but with a slight difference in the slope. As we analyzed in the previous section and show in Figure 7, this is because the spectrum slope is related to $\epsilon_{B}$ at $\mathrm{keV}$ energies, where the synchrotron radiation of cascaded electrons dominates. The value of $\epsilon_{B}$ in the case of $\gamma_{p, \max }^{\prime}=10^{5}$ is smaller than that in the case of $\gamma_{p, \max }=10^{6}$, so the spectrum at $\mathrm{keV}$ energies in the latter case is softer than that in the former case.

The narrow Band component in this GRB may imply the photospheric emission (Zhang et al. 2011). In this case, the emission radius may be smaller than what we used for fitting. However, when we adopt a smaller radius and larger $\Gamma$ (to avoid a too-strong $\gamma \gamma$ absorption), the low-energy spectrum at $\sim 10 \mathrm{keV}$ becomes too hard to agree with the observed lowenergy excess.
We note our fitting result in a large baryonic loading factor of $\epsilon_{p}>100$, while in Asano et al. (2010), they adopted $\epsilon_{p}=3$ only. One reason for such a difference is that their result did not really reach the flux level of the observed one above $\sim \mathrm{GeV}$. Another reason is that they used a very high maximum proton energy estimated in the Bohm limit, which enhances the overall efficiency for the photomeson process.

\subsection{GRB 080916C}

GRB 080916C is the most powerful GRB ever recorded with the highest inferred isotropic energy, $E_{\text {iso }}=8.8 \times 10^{54} \mathrm{erg}$, in the energy range of $10 \mathrm{keV}-10 \mathrm{GeV}$, located at a large redshift, $z=4.35$ (Abdo et al. 2009b). The time-integrated spectrum between $T_{0}+3.58$ and $T_{0}+7.68 \mathrm{~s}$, i.e., the interval " $\mathrm{b}$ " in Abdo et al. (2009b), is fitted by a single Band function with $\alpha=-1.02, \beta=-2.21$, and $\varepsilon_{\gamma, p}=1170 \mathrm{keV}$ from $\mathrm{keV}$ to $\mathrm{GeV}$. A correlation in temporal behavior between the GBM and LAT emission is found in this GRB as well. Although a single Band function seems to explain the entire spectrum of this GRB from $\mathrm{keV}$ to $\mathrm{GeV}$, the poor statistics at $\mathrm{GeV}$ cannot rule out a different origin for the $\mathrm{GeV}$ emission from the $\mathrm{keV} / \mathrm{MeV}$ emission.

Similar to the treatment for the two bursts above, we consider the cases for $\gamma_{p, \text { max }}^{\prime}=10^{5}$ and $\gamma_{p \text {, max }}=10^{6}$, and the two corresponding Bohm factors are $\mathcal{A} \simeq 730$ and $\mathcal{A} \simeq 73$ under two groups of parameters adopted in Figure 10, respectively. Although the soft spectrum at high energy may be consistent with a synchrotron origin, the value of $\epsilon_{B}$ or magnetic field for this burst cannot be too large, otherwise the strong synchrotron radiation of the secondary $e^{ \pm}$will lead to a low-energy excess $\lesssim 10 \mathrm{keV}$, which is not observed. Thus, we expect that IC radiation of secondary electrons is the main contributor of high-energy photons. By treating the observed photons as the target radiation field, the $\gamma \gamma$-annihilation opacity for the highest-energy observed photon implies a minimum bulk Lorentz factor of $\Gamma_{\min } \sim 900$ and a dissipation radius of $R \gtrsim 10^{15} \mathrm{~cm}$ for the interval considered (Abdo et al. 2009b; Zhang \& Peer 2009). In our calculations, $\Gamma=1500$ and $R=10^{15} \mathrm{~cm}$ are employed for the adopted $\gamma_{p, \max }^{\prime}$. Such a large bulk Lorentz factor and a large dissipation radius yield a low 

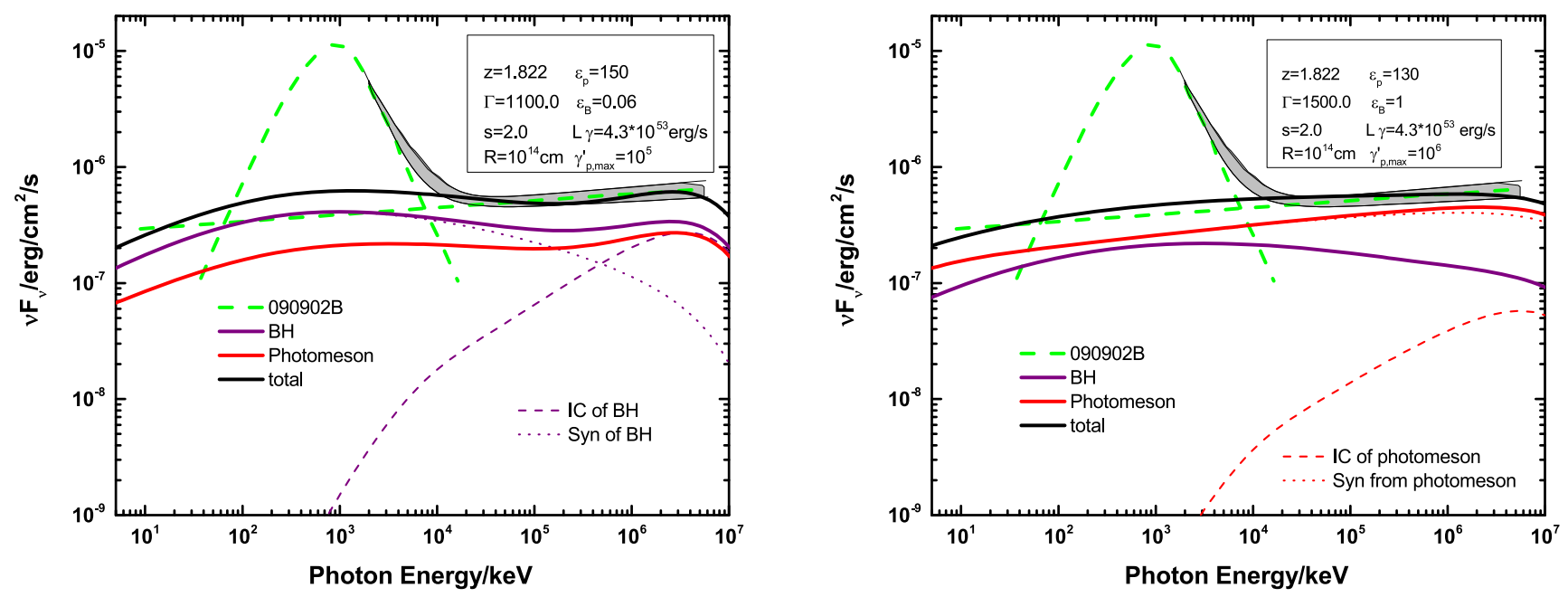

Figure 9. Same as Figure 8, but for GRB 090902B.

efficiency for the interactions of protons, so a large proton energy budget is needed, as in the case of GRB 090902B (Wang et al. 2009). The results are shown in Figure 10.

Given a large break energy $\varepsilon_{\gamma, p}=1170 \mathrm{keV}$ and a hard spectrum after the break $(\beta=-2.21)$, the photomeson process has more contribution than the $\mathrm{BH}$ process around $\mathrm{GeV}$, even in the case of $\gamma_{p \text {, max }}^{\prime}=10^{5}$. For different maximum proton energies, the photomeson process contributes more around $\mathrm{GeV}$ than the $\mathrm{BH}$ process. The flux of the cascade emission peaks around $\mathrm{GeV}$, and the flux level is consistent with the extrapolation of the Band component from $\mathrm{MeV}$.

\section{Discussions}

\subsection{Synchrotron self-Compton Radiation of Primary Electrons}

In our calculation, we neglect the possible contribution by primary electrons to high-energy flux. In principle, we can expect electrons are accelerated to relativistic energies in a GRB jet and scatter off photons from the Band component up to the $\mathrm{GeV}-\mathrm{TeV}$ range, although the Klein-Nishina effect can suppress the flux to a certain extent. In an early study (Gupta \& Zhang 2007), the authors compared the hadronic emission and the Synchrotron self-Compton (SSC) emission by primary electrons in GRB jets within different sets of parameter regimes, and they found that the SSC emission could outshine the hadronic emission under certain conditions. Here we check the SSC contribution of the primarily accelerated electrons under the adopted parameters in the above fittings.

The synchrotron radiation of nonthermal electrons may contribute flux at the $\mathrm{keV} / \mathrm{MeV}$ range. Although the synchrotron origin of the Band component suffers from a lot of criticisms (see Zhang 2011; Zhang et al. 2016 for reviews), we normalize the synchrotron emission to the observed Band component here to obtain an upper limit for the amount of the primarily accelerated electrons, regardless of the excess at low energy of GRB 090902B and GRB 090926A. We then calculate the SSC radiation from the obtained electrons and the initiated cascade emission following the same treatment to the hadronic one. Due to the Klein-Nishina effect, SSC emission is severely suppressed at high energy, which causes the spectrum break in the SSC component shown in Figure 11. Note that the cascade spectrum is more or less universal for a given background photon field, as long as the cascade is initiated at a sufficiently high energy and fully developed. Thus, whether the proton-initiated cascade emission (i.e., hadronic emission) or the SSC-initiated cascade emission dominates is determined by the separate injection rate. For GRB 090902B, given a large $\epsilon_{B}$ and a large $\epsilon_{p}$, we expect that the SSC-initiated cascade emission is much weaker than the proton-initiated cascade emission, which can also be seen in the figure. For GRB 080916C, the $\epsilon_{B}$ is much smaller, which leads to a higher SSC intensity, but the $\epsilon_{p}$ is still large enough so that the hadronic emission still dominates. On the contrary, we have a small $\epsilon_{B}$ and a (relatively) small $\epsilon_{p}$ for GRB 090926A. As a result, the SSC-initiated cascade exceeds the hadronic one. Nevertheless, this does not rule out the hadronic origin of the high-energy emission of GRB 090926A, because the result is based on the assumption that the Band component is synchrotron emission. For GRB 090926A, the low-energy photon index is harder than the so-called "line of death" of the synchrotron radiation (i.e., $-2 / 3$; Preece et al. 1998), implying some other origin, e.g., photospheric radiation and Comptonization (Medvedev 2000; Mészáros \& Rees 2000; Peer et al. 2012). Nevertheless, there may still be some electrons being accelerated along with protons to a power-law distribution in GRB 090926A. We also check the emission of such a component of electrons. By using the same Bohm parameter $\mathcal{A}$ and power-law index as for protons and normalizing the total electron luminosity by $L_{e}=L_{\gamma}$ (or the energy equipartition coefficient for electrons is $\epsilon_{e} \equiv U_{e} / U_{\gamma}=1$, with $U_{e}$ the energy density of nonthermal electrons in the comoving frame), we obtain an electron spectrum and cascade emission, as shown with the blue solid curve in the leftmost panel of Figure 11. To make the electron contribution less important than the hadronic one for the adopted parameters in GRB 090926A, $\epsilon_{e}<1$ (or $\left.L_{e} / L_{p}<1 / 15\right)$ is required. Such a result is fairly expected, since electrons are more efficient emitters than protons, and it is consistent with what was found in the previous study (Gupta \& Zhang 2007).

\subsection{Neutrino Emission}

Since the photomeson process produces high-energy neutrinos via the decay of charged pions and muons, GRBs have been considered as one of the most promising high-energy neutrino sources. However, no significant correlation between high-energy neutrino events and GRB events has been detected 

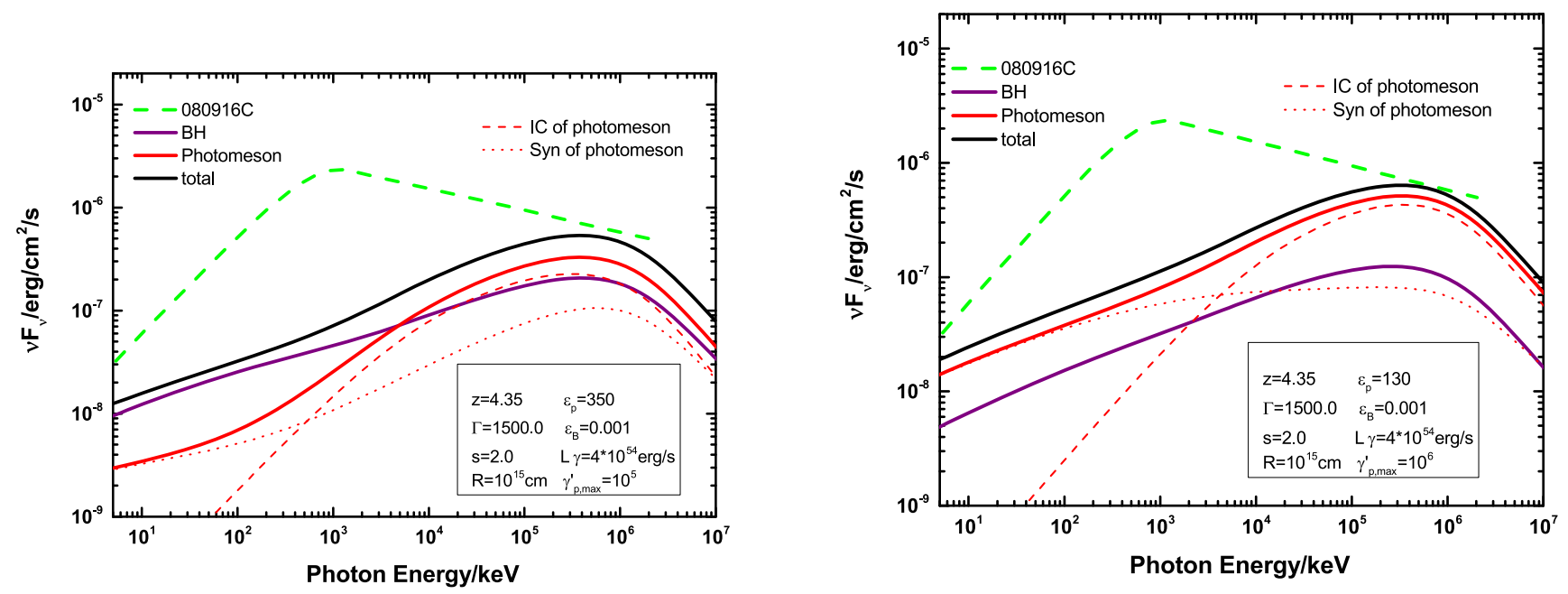

Figure 10. Same as Figure 10, but for GRB 080916C.

by the IceCube neutrino telescope. This result indicates a low neutrino emission rate of GRB jets, due to either a low photomeson efficiency or a low baryonic loading factor, and hence puts stringent constraints on the parameters of GRB jets, e.g., the baryonic loading factor $\epsilon_{p}$, the bulk Lorentz factor $\Gamma$, and the dissipation radius $R$ (Aartsen et al. 2015, 2016, 2017). Note that the parameters adopted in our fittings to GRB 090926A, GRB 09092B, and GRB 080916C are in the allowed region. In our fittings, we adopt two maximum proton energies, i.e., $\gamma_{p, \max }^{\prime}=10^{6}$ and $\gamma_{p, \max }^{\prime}=10^{5}$. In the former case, the high-energy prompt emission mainly arises from the photomeson process, and the peak flux of the neutrinos is comparable to that of the photons, as expected from the branching ratio of the photomeson process. In the case of $\gamma_{p, \max }^{\prime}=10^{5}$, the $\mathrm{BH}$ process may dominate the high-energy prompt emission, and hence the neutrino flux that is related to the photomeson process is lower than the high-energy photon flux. Also, due to a lower maximum proton energy, the neutrino flux peaks at a lower energy. On the other hand, the overall interaction efficiency in the case of $\gamma_{p \text {, max }}^{\prime}=10^{5}$ is smaller than that in the case of $\gamma_{p \text {,max }}^{\prime}=10^{6}$, so the required baryonic loading factor in the former case is larger than that in the latter case. Thus, the neutrino fluxes in the two cases, as shown in Figure 12, result in a similar neutrino detection probability by IceCube. Given the effective area of IceCube and the zenith angle of the GRB, and assuming a flavor ratio of 1:1:1 after oscillation, we find that the probability of detecting a muon neutrino or anti-muon neutrino in the range of $10 \mathrm{TeV}-10 \mathrm{PeV}$ is 0.001 for GRB 090926A, 0.03 for GRB 090902B in both cases of $\gamma_{p, \max }^{\prime}$, and 0.01 in the case of $\gamma_{p, \max }^{\prime}=10^{5}$ and 0.008 in the case of $\gamma_{p, \max }^{\prime}=10^{6}$ for GRB 080916C. Provided that 10 GRBs of similar properties to these three kinds of GRBs are detected by the LAT per year, we expect $<1$ GRB-correlated neutrinos can be detected for a period of $9 \mathrm{yr}$ (2008-2017), which is consistent with the observation. But the persistent accumulative neutrino flux from the future LAT GRBs would lead to the detection of a correlation between the neutrino event and the LAT GRBs in another $\sim 10 \mathrm{yr}$. The detection or nondetection of neutrinos for LAT GRBs in the future could be a test for the hadronic model. However, for the majority of GRBs, we expect a quite different set of parameters, especially a much smaller baryon loading factor, which should basically follow the constraint from the nondetection of GRB-neutrino correlation by IceCube (Aartsen et al. 2015, 2016, 2017). This was also found from early theoretical studies (He et al. 2012; Li 2012, 2013; Liu \& Wang 2013; Zhang \& Kumar 2013).

We also note that the constraints on GRB jet parameters from IceCube are based on the assumption that $\mathrm{A} \sim 1$, which usually leads to $\gamma_{p \text {, max }} \sim 10^{8}-10^{9}$. However, for most GRBs in which high-energy emissions are not detected, we do not need to invoke such a large maximum proton energy to explain the $\mathrm{GeV}$ emission with the hadronic model. Thus, the maximum proton energy or Lorentz factor could be as low as $10^{4}$. In Figure 13, we present the neutrino flux from a typical GRB of $L_{\gamma}=10^{51} \mathrm{erg} \mathrm{s}^{-1}$ under different maximum proton energy, with all other parameters the same as the benchmark parameters. Because the photomeson efficiency drastically decreases with the proton energy, we can see both the peak energy and the peak flux of the neutrino decrease with decreasing $\gamma_{p \text {,max }}^{\prime}$. Comparing the neutrino flux in the case of $\gamma_{p, \max }^{\prime}=10^{7}$ (corresponding to $\mathcal{A} \simeq 220$ ) to the case of $\gamma_{p, \max }^{\prime}=10^{4}$ (corresponding to $\mathcal{A} \simeq 9.2 \times 10^{5}$ ), the peak flux is two orders of magnitude lower than the former one. And the predicted neutrino event in the latter case is less than that in the former case by more than one order of magnitude. Thus, with a low maximum proton energy, the current constraints on GRB parameters such as the baryonic loading factor and bulk Lorentz factor could be relaxed to a certain extent.

\subsection{Low-energy Excess Due to Cascade Emission}

The synchrotron radiation of the secondary $e^{ \pm}$may lead to a low-energy excess in the spectrum if the low-energy spectrum of the Band component is hard (i.e., a large $\alpha$ ), such as in GRB 090902B. This feature could be an indication of the baryon component in the composition of a GRB jet (Asano et al. 2010). However, for most GRBs, the low-energy Band spectrum is not hard enough that the Band component would conceal such a component above the threshold energy of the current detectors (e.g., Fermi-GBM at $8 \mathrm{keV}$ ), even it really exists. Future GRB detectors, such as the SVOM satellite (Cordier et al. 2015), have a lower threshold energy of $4 \mathrm{keV}$ and thus provide a better chance to reveal such a low-energy excess. If the synchrotron radiation extends to an even lower 

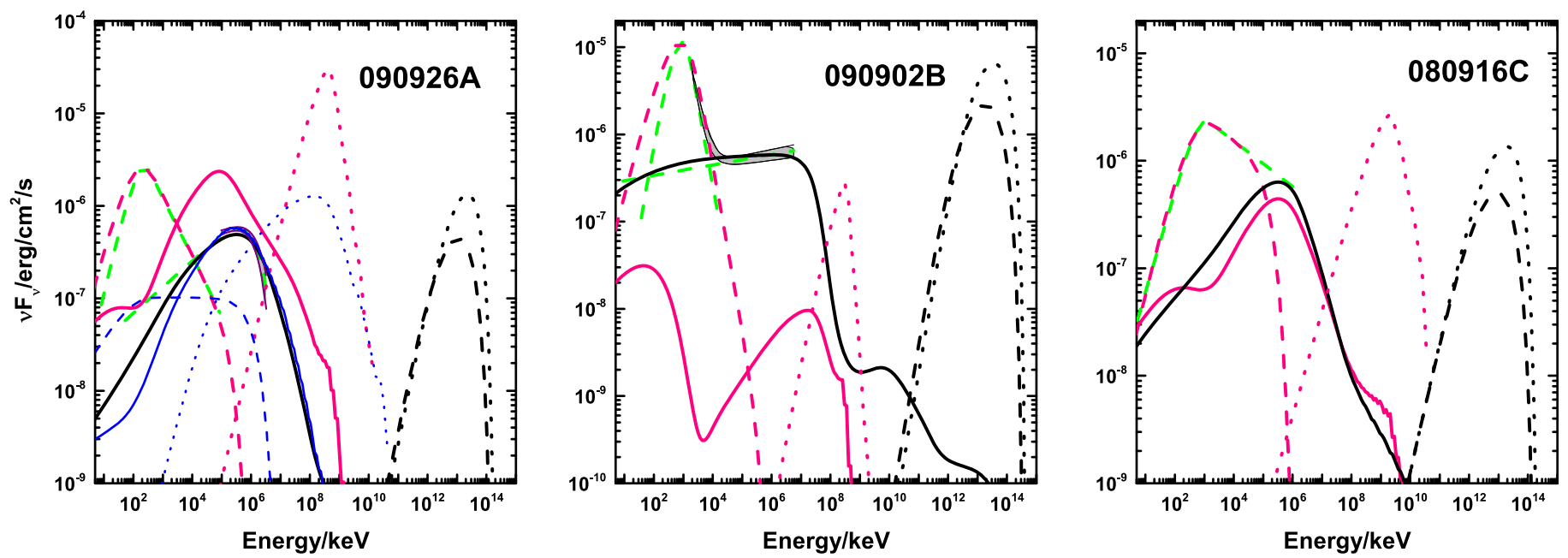

Figure 11. Cascade emissions initiated by SSC emissions of primary electrons for GRBs 090902B, 090926A, and 080916C (pink solid curves) by normalizing the corresponding synchrotron emission (pink dashed curves) with the Band component (see discussion in Section 4.1). The unabsorbed SSC emissions are shown as pink dotted curves. The injection rate of the first-generation secondary photons and electrons from the photomeson process are shown as black dotted and black dashed curves, respectively, while their initiated cascade emissions in the quasi-steady state are shown as black solid curves. Note that the components shown with pink dotted curves, black dotted curves, and black dashed curves cannot be observed, since they will have interactions and initiated EM cascade inside the GRB jet. They are presented here just to show the injection rate for the respective cascades. In all three panels, the parameters of the GRBs are exactly same as those used in the bottom panels of Figures 8-10. Some additional parameters for the primary electron distributions, i.e., the break Lorentz factors in the electron spectrum, the low-energy spectral slope, and the high-energy spectral slope, are set to 4883, 0.82, and -4.3 for GRB 090926A; 1643, 2.14, and -6.80 for GRB 090902B; and 2.21 × 10 ${ }^{4}, 0.04$, and -3.42 for GRB 080916C, respectively. The blue solid curve in the leftmost panel represents the cascade emission from a coaccelerated electron component with $\epsilon_{e}=1$. See the text for details.

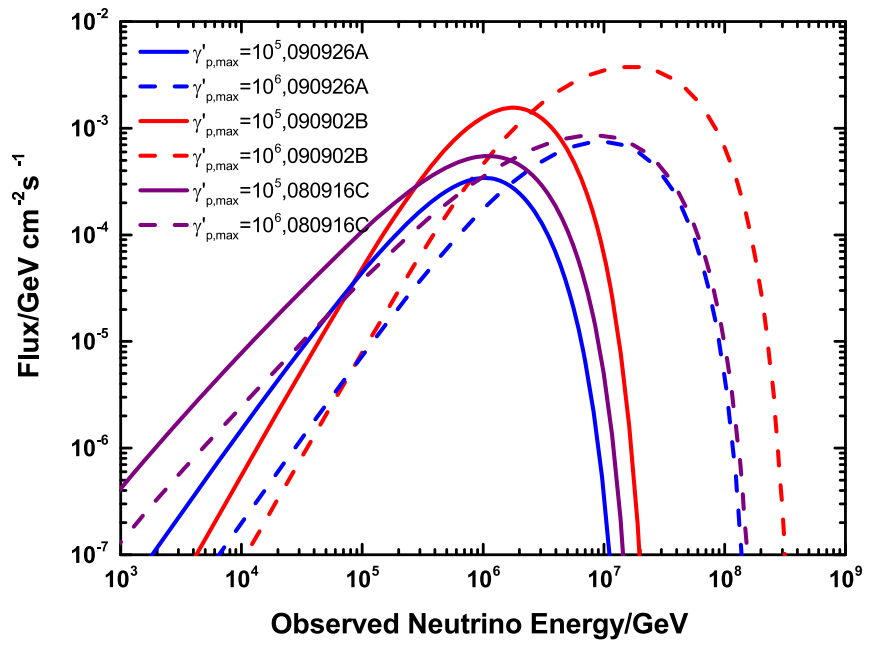

Figure 12. All-flavor neutrino flux for GRB 090926A (blue), GRB 090902B (red), and GRB 080916C (purple), obtained under the same parameters employed in Figures 8, 9, and 10, respectively.

energy, such as the optical band, there may be a chance to capture it with the GWAC, which is the ground-based system of the SVOM mission. But we should be cautious, because the synchrotron radiation of the secondary $e^{ \pm}$may undergo selfabsorption below a certain energy, and the optical emission of GRBs may stem from some other mechanisms.

\section{Summary}

To summarize, we revisited the hadronic model for the highenergy emission of GRBs in the prompt emission phase. While the energy of accelerated protons in GRB jets is assumed to reach $\sim 10^{20} \mathrm{eV}$ in the literature, we show that, in the case of a lower maximum proton energy, the photomeson process may be less important than the $\mathrm{BH}$ process in converting energies of

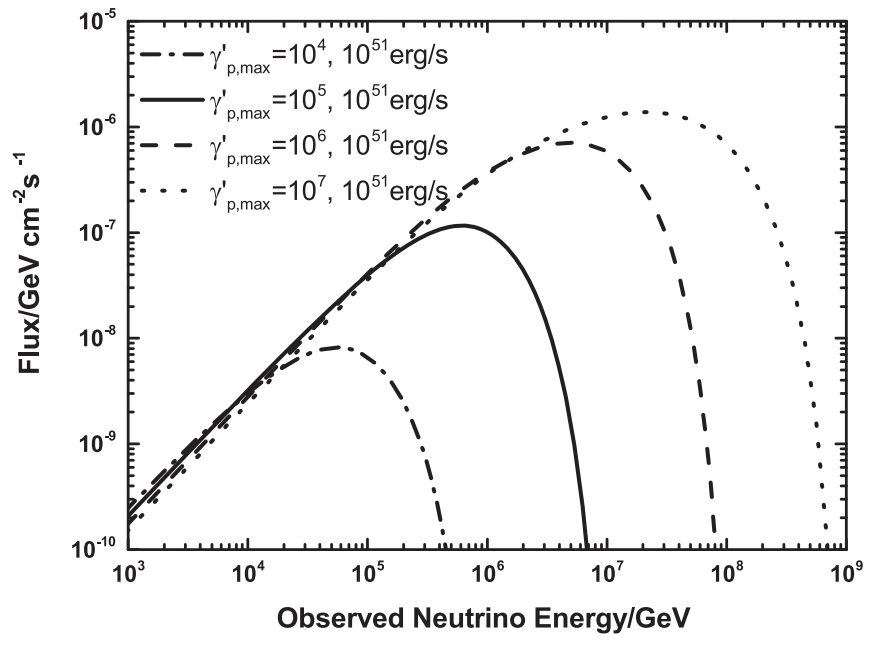

Figure 13. All-flavor neutrino flux from a GRB of the typical luminosity $L_{\gamma}=10^{51} \mathrm{erg} \mathrm{s}^{-1}$ (in $1 \mathrm{keV}-10 \mathrm{MeV}$ ) with a different maximum proton energy (Lorentz factor) in the comoving. Other parameters are the same as the benchmark parameters listed in Table 1 .

protons to gamma rays via an EM cascade. Taking GRB 090926A, GRB 090902B, and GRB 080916C as examples, we found that the synchrotron radiation and IC scattering of the secondary $e^{ \pm}$from the proton-induced cascade via the $\mathrm{BH}$ and photomeson processes can reproduce various types of the observed spectrum of the prompt high-energy emission of GRBs with a relatively low maximum proton energy. The adopted parameters in the spectrum fittings are consistent with the constraints from the null detection of GRB-correlated neutrino events by the IceCube neutrino telescope. The cascade emission may also lead to a low-energy excess below a few $\mathrm{keV}$ and might be used as an indication of the baryon component in the GRB ejecta. 
We thank Prof. Felix Aharonian for his invaluable discussion and the anonymous referee for the useful comments. This work is supported by the National Basic Research Program ("973" Program) of China (grant No. 2014CB845800), the National Key Research and Development Program of China (grant No. 2017YFA0402600), and the National Natural Science Foundation of China (grant No. 11573014). This work is also partly supported by the joint research program of the Institute for Cosmic Ray Research (ICRR), the University of Tokyo, and Grants-in-Aid for Scientific Research Nos. 15K05069 and 16K05291 (K.A.) from the Ministry of Education, Culture, Sports, Science and Technology (MEXT) of Japan.

\section{Appendix \\ Solving the Spectrum of Cascaded Electrons in the Quasi-steady State}

Basically, we follow the treatment in Böttcher et al. (2013) for blazars but also consider the additional $\mathrm{BH}$ process for protons and IC radiation for electrons, which are important in the scenario of GRB jets. The key to obtaining the cascaded electron spectrum in the quasi-steady state is to solve the following equation:

$$
n_{e}^{\prime}\left(\gamma_{e}^{\prime}\right)=-\frac{1}{\dot{\gamma}_{e}^{\prime}} \int_{\gamma_{e}^{\prime}}^{\infty} d \tilde{\gamma}_{e}^{\prime}\left[Q_{e}\left(\tilde{\gamma}_{e}^{\prime}\right)+\dot{n}_{e, \gamma \gamma}^{\prime}\left(\tilde{\gamma}_{e}^{\prime}\right)\right]
$$

The cooling rate of electrons via synchrotron radiation and IC scattering can be given by

$$
\dot{\gamma}_{e}^{\prime}=-\frac{c \sigma_{T} B^{\prime 2}}{6 \pi m_{e} c^{2}} \gamma_{e}^{\prime 2}+\dot{\gamma}_{e, \mathrm{IC}}^{\prime},
$$

where $\dot{\gamma}_{e, \text { IC }}^{\prime}$ accounts for the IC cooling, including the KleinNishina effect, which can be calculated following Blumenthal $\&$ Gould (1970). Here $Q_{e}\left(\gamma_{e}^{\prime}\right)$ is the injection rate of the firstgeneration electrons (including both $e^{-}$and $e^{+}$) from the photomeson and $\mathrm{BH}$ processes, which are calculated based on the semi-analytical treatment in Kelner \& Aharonian (2008). Also, $\dot{n}_{e, \gamma \gamma}^{\prime}\left(\tilde{\gamma}_{e}^{\prime}\right)$ is the pair production rate through the $\gamma \gamma$ annihilation, including the annihilation of the high-energy photon from the neutral pion decay produced in the photomeson process and the high-energy photon produced by the synchrotron radiation and IC scattering, i.e.,

$$
\begin{aligned}
\dot{n}_{e, \gamma \gamma}^{\prime}\left(\gamma_{e}^{\prime}\right)= & f_{\mathrm{abs}}\left(\varepsilon_{1}\right)\left(\dot{n}_{\varepsilon_{1}}^{0}+\dot{n}_{\varepsilon_{1}}^{\mathrm{sy}}+\dot{n}_{\varepsilon_{1}}^{\mathrm{IC}}\right) \\
& +f_{\mathrm{abs}}\left(\varepsilon_{2}\right)\left(\dot{n}_{\varepsilon_{2}}^{0}+\dot{n}_{\varepsilon_{2}}^{\mathrm{sy}}+\dot{n}_{\varepsilon_{2}}^{\mathrm{IC}}\right),
\end{aligned}
$$

with

$$
f_{\mathrm{abs}}(\varepsilon)=1-\frac{1-e^{-\tau_{\gamma \gamma}(\varepsilon)}}{\tau_{\gamma \gamma}(\varepsilon)}
$$

being the absorbed fraction of photons. Equation (6) contains two parts, since the $\gamma \gamma$ annihilation produces two electrons, taking a fraction $f_{\gamma}$ and $1-f_{\gamma}$ of the energy of the initial highenergy photon, respectively. If the center-of-mass energy of the interaction is sufficiently high, one of the outgoing electrons will take most of the energy of the initial photon. According to Böttcher et al. (2013), $f_{\gamma}=0.9$ is in good agreement with the numerical Monte Carlo simulations. As a result, to produce an electron with energy $\gamma_{e}^{\prime}$, the photons should have the energy of either $\varepsilon_{1}=\gamma_{e}^{\prime} / f_{\gamma}$ or $\varepsilon_{2}=\gamma_{e}^{\prime} /\left(1-f_{\gamma}\right)$. In Equation (6), $\dot{n}_{\varepsilon}^{0}$ can also be found by the analytical treatment in Kelner \& Aharonian (2008). Here $\dot{n}_{\varepsilon}^{\text {sy }}$ can be described as

$$
\dot{n}_{\varepsilon}^{\text {sy }}=A_{0} \varepsilon^{-2 / 3} \int_{1}^{\infty} d \gamma_{e}^{\prime} n_{e}^{\prime}\left(\gamma_{e}^{\prime}\right) \gamma_{e}^{\prime-2 / 3} e^{-\varepsilon /\left(b \gamma_{e}^{\prime 2}\right)},
$$

with

$$
A_{0}=\frac{c \sigma_{T} B^{\prime 2}}{6 \pi m_{e} c^{2}} \frac{1}{\Gamma(4 / 3) b^{4 / 3}},
$$

where $\Gamma(4 / 3)=0.89297, b=B^{\prime} / B_{\text {crit }}$, and $B_{\text {crit }}=4.4 \times$ $10^{13} \mathrm{G}$, while $\dot{n}_{\varepsilon}^{\text {IC }}$ can be given by

$$
\dot{n}_{\varepsilon}^{\mathrm{IC}}=\int_{1}^{\infty} d \gamma_{e}^{\prime} n_{e}^{\prime}\left(\gamma_{e}^{\prime}\right) \frac{1}{\gamma_{e}^{\prime} m_{e} c^{2}} \frac{d N}{d t d E_{1}},
$$

where $\frac{d N}{d t d E_{1}}$ is given by Equation (2.48) of Blumenthal \& Gould (1970).

Since the electron spectrum $n_{e}^{\prime}\left(\gamma_{e}^{\prime}\right)$ exists on both sides of Equation (4), this equation can be evaluated progressively, starting from the highest electron energies and then using the solution of $n_{e}^{\prime}\left(\gamma_{e}^{\prime}\right)$ for large $\gamma_{e}^{\prime}$ as one progresses toward the lower values of $\gamma_{e}^{\prime}$, to obtain the equilibrium pair distribution $n_{e}^{\prime}\left(\gamma_{e}^{\prime}\right)$, which has an excellent agreement with the results of the Monte Carlo simulations (Böttcher et al. 2013). Then, one can obtain the synchrotron and IC spectra from the equilibrium pair distribution after the absorption by the target photon field. Since the characteristic energy for the absorption of $\mathrm{GeV}$ photons by photon-photon annihilation is around tens of $\mathrm{MeV}$, where the contribution of the extra component could be higher than that of the Band component, to get the final and correct spectra of photons, we execute an iteration procedure until the self-consistent results after the photon-photon absorption are reached.

\section{ORCID iDs}

Ruo-Yu Liu (ib https://orcid.org/0000-0003-1576-0961 Zi-Gao Dai (iD https://orcid.org/0000-0002-7835-8585 Katsuaki Asano (iD https://orcid.org/0000-0001-9064-160X

\section{References}

Aartsen, M., Abraham, K., Ackermann, M., et al. 2016, ApJ, 824, 115 Aartsen, M., Ackermann, M., Adams, J., et al. 2015, ApJL, 805, L5 Aartsen, M. G., Ackermann, M., Adams, J., et al. 2017, ApJ, 843, 112 Abdo, A. A., Ackermann, M., Ajello, M., et al. 2009a, ApJL, 706, L138 Abdo, A. A., Ackermann, M., Arimoto, M., et al. 2009b, Sci, 323, 1688 Ackermann, M., Ajello, M., Asano, K., et al. 2011, ApJ, 729, 114 Ackermann, M., Ajello, M., Asano, K., et al. 2013a, ApJS, 209, 11 Ackermann, M., Ajello, M., Asano, K., et al. 2013b, ApJ, 763, 71 Asano, K. 2005, ApJ, 623, 967

Asano, K., Guiriec, S., \& Mészáros, P. 2009a, ApJL, 705, L191 Asano, K., Inoue, S., \& Mészáros, P. 2009b, ApJ, 699, 953 Asano, K., Inoue, S., \& Mészáros, P. 2010, ApJL, 725, L125 Band, D., Matteson, J., Ford, L., et al. 1993, ApJ, 413, 281 Beloborodov, A. M., Hascoët, R., \& Vurm, I. 2014, ApJ, 788, 36 Blumenthal, G. R., \& Gould, R. J. 1970, RvMP, 42, 237

Böttcher, M., Reimer, A., Sweeney, K., \& Prakash, A. 2013, ApJ, 768, 54 Chodorowski, M. J., Zdziarski, A. A., \& Sikora, M. 1992, ApJ, 400, 181 Cordier, B., Wei, J., Atteia, J.-L., et al. 2015, arXiv:1512.03323 Crumley, P., \& Kumar, P. 2013, MNRAS, 429, 3238

Fukushima, T., To, S., Asano, K., \& Fujita, Y. 2017, ApJ, 844, 92 Gao, S., Asano, K., \& Mészáros, P. 2012, JCAP, 11, 058

Guiriec, S., Kouveliotou, C., Daigne, F., et al. 2015, ApJ, 807, 148 Gupta, N., \& Zhang, B. 2007, MNRAS, 380, 78

He, H. N., Liu, R. Y., Wang, X. Y., et al. 2012, ApJ, 752, 29

Kelner, S. R., \& Aharonian, F. A. 2008, PhRvD, 78, 034013 
Kumar, P., \& Barniol Duran, R. 2009, MNRAS, 400, L75

Kumar, P., \& Barniol Duran, R. 2010, MNRAS, 409, 226

Kumar, P., \& Zhang, B. 2015, PhR, 561, 1

Li, Z. 2012, PhRvD, 85, 027301

Li, Z. 2013, ApJL, 770, L40

Liu, R.-Y., \& Wang, X.-Y. 2013, ApJ, 766, 73

Liu, R.-Y., Wang, X.-Y., \& Wu, X.-F. 2013, ApJL, 773, L20

Maxham, A., Zhang, B.-B., \& Zhang, B. 2011, MNRAS, 415, 77

Medvedev, M. V. 2000, ApJ, 540, 704

Mészáros, P., \& Rees, M. J. 2000, ApJ, 530, 1

Mücke, A., Rachen, J. P., Engel, R., Protheroe, R. J., \& Stanev, T. 2000, NuPhS, 80, 08
Peer, A., Zhang, B.-B., Ryde, F., et al. 2012, MNRAS, 420, 468

Preece, R. D., Briggs, M. S., Mallozzi, R. S., et al. 1998, ApJL, 506, L23

Rees, M. J., \& Mészáros, P. 2005, ApJ, 628, 847

Tang, Q.-W., Wang, X.-Y., \& Liu, R.-Y. 2017, ApJ, 844, 56

Wang, X. Y., Li, Z., Dai, Z. G., \& Mészáros, P. 2009, ApJL, 698, L98

Wang, X.-Y., Liu, R.-Y., \& Lemoine, M. 2013, ApJL, 771, L33

Zhang, B. 2011, CRPhy, 12, 206

Zhang, B. B., Zhang, B., Liang, E. W., et al. 2011, ApJ, 730, 14

Zhang, B., \& Kumar, P. 2013, PhRvL, 110, 121101

Zhang, B., \& Peer, A. 2009, ApJL, 700, L65

Zhang, B., \& Yan, H. 2011, ApJ, 726, 90

Zhang, B., Lu, H. J., \& Liang, E. W. 2016, SSRv, 202, 3 